Aus Dr. Max Joseph's Poliklinik für Eautkrankheiten in Berlin.

\title{
Beiträge zur Kenntniss der Plasmazellen, insbesondere beim Lupus.
}

\author{
Von \\ Dr. Johan Almkvist \\ in Stockholn. \\ (Hiezu Taf. VII u. VIII.)
}

Im Jahre 1891 lenkte Un n a die Aufmerksamkeit auf die von ihm entdeckten sogenannten Plasmazellen. Er beschrieb (18) das Vorkommen und Aussehen dieser Zellen im Lupusgewebe. Seitdem ist eine grosse Literatur über diesen Gegenstand theils von Un na selbst (17), (19), theils von vielen anderen Beobachtern erschienen. $\mathrm{J}$ a dassohn war der erste, der sich gegen Unna's Beschreibung und Anschauung wandte (4). So entspann sich zwischen Ja da s s o hn (5), (6) und Unna (20) eine Polemik, die später zwischen Unna (21) und v. M a r s chalk fortgesetzt wurde, nachdem v. Marschalko in seiner 1895 erschienenen Arbeit über diese Zellen (11) zu einer ganz entgegengesetzten Ansicht als Unna gekommen war. An diesem Streit nahm auch Neisser mit einer Kritik über Unna Theil (14), (15). Seit 1895 haben ziemlich viele Autoren z. B. Hodara (3), Schottländer (16), Justi (8) und Krompecher (9) über diese Plasmazellen gearbeitet. Im Allgemeinen haben sie v. Marschalko's Untersuchungen mehr oder weniger bestätigt. Dies veranlasste v. Marschalko im Jahre 1899 ein Resumé über die Plasmazellenfrage zu geben (12), in welcher er die Plasmazellenfrage als beinahe erledigt erklärt und glaubt, dass nur wenig "von der ganzen Entdeckung Unn a's" übrig geblieben ist. Bald darauf erschien aus Paltauf's Institut in Wien eine Arbeit 
von Joannovics (7), die allerdings nicht mit v. Marschalko's Ansicht übereinstimmte. Marschalko hat dann über Joannovies Arbeit eine Kritik geschrieben (13), in welcher er die Behauptungen von Joannovics zu widerlegen versucht. Ihm erscheint - sagt er - „eine Revision der ganzen Plasmazellenlehre ganz überflüssig".

Leider kann ich dieser Meinung von Marschalko's, dass der Erklärungsprocess in der Plasmazellenfrage beinahe schon zu Ende geführt ist, nicht beistimmen. Ich finde im Gegentheil, dass nur in einem Punkt die verschiedenen Autoren vollkommen übereinstimmen, nämlich in dem Vorkommen der Plasmazellen bei ziemlich vielen pathologischen Processen. In allen übrigen Punkten dieser Frage sind wir, wie es mir scheint, von der Lösung mehr oder weniger weit entfernt.

Da ich der Meinung bin, dass diese Frage eine sehr wichtige nicht nur für die Dermatologie, sondern auch und vielleicht noch mehr für die allgemeine Pathologie und die Zellenlehre ist, nahm ich mit grosser Freude den Vorschlag von Herrn Dr. Max Joseph in Berlin an, das in seinem Laboratorium befindliche Lupusmaterial besonders mit Rücksicht auf die Plasmazellen zu untersuchen.

Das Material, welches er zu meiner Disposition stellte, bestand aus in Alkohol gehärteten und in Celloidin eingebetteten Schnitten von $21 \mathrm{Krankheitsfällen.} \mathrm{Von} \mathrm{diesen} \mathrm{waren} 15$ als Lupus, 5 als Tuberculosis cutis verrucosa klinisch sicher diagnosticirt. Dazu kam eine tuberculöse Lymphdrüse.

Die Schnitte wurden hauptsächlich mit Unna's polychromer Methylenblau-Färbemethode mit Entfärbung in Glycerinäthermischung behandelt. Daneben wurden auch Schnitte in gewöhnlichem Methylenblau, Thionin, Kresylviolett, Methylgrün, Biondi-Heidenhains Farbenmischung, saurem Orcein, Weigert's Resorcin-Fuchsin, Eosin, Saffranin und Hämatoxylin gefärbt.

Ich will zunächst die interessanteren Fälle, die ein charakteristisches Bild zeigen, ausführlicher besprechen und die anderen dann kürzer erwähnen.

Fall 1. Lupus vulgaris faciei. Das Präparat zeigt einen gut ausgebildeten Herd, in dessen mittlerem Theil die Epidermis zerstört, in den Seitentheilen dagegen ziemlich unverändert ist. Der Herd besteht aus einer Anzahl tuberkelähnlicher Knötchen mit einem runden hellen, 
schwach gefärbten Centrum und um dieses herum eine ringförmige Zone von dichtliegenden, stärker gefärbten Zellen. Da, wo das Epithelgewebe zerstört ist, liegen die Knötchen unbedeckt und hier liegen sie auch unmittelbar neben einander, so dass dieser Theil den Eindruck macht, der am meisten veränderte und also auch älteste zu sein. In Uebereinstimmung hiermit zeigen auch die Färbungen mit saurem Orcein und Weigerts Resorcin-Fuchsin in diesem Theil des Präparates gar keine elastische Fasern. Peripheriewärts hiervon liegen die Knötchen nicht so dicht, sondern baben zwischen sich mebr oder weniger zellig infiltrirtes Bindegewebe, wo auch einige Reste von dem elastischen Fasernetz noch vorhanden sind. Wo keine Knötchen sind, sieht man die elastischen Fasern in vollkommen normaler Weise ausgebildet. Der vermehrte Zellreichthum des Bindegewebes erstreckt sich auch in das subcutane Fettgewebe hinein, so dass man zwischen den Fettzellen zahlreichere und grössere Zellen als normal sieht. Ziemlich weit in das Fettgewebe hinein findet man hier und da sehr kleine Knötchen von blassen Zellen mit einer sehr schwach ausgeprägten Zone von stärker tingirten Zellen umgeben. Blutgefässe kommen ziemlich häufig zwischen den Knötchen vor. Ganz kleine findet man auch in den Knötchen und in den Septen des Fettgewebes.

Histologisch entspricht also dieser Fall der Form, die Unna als circumscript oder nodulär bezeichnet hat.

Wenn wir dann die feineren Details dieses Präparates näher studiren, so sehen wir, dass die blassen Centren der Knötchen aus ziemlich grossen, polymorph geformten, sehr schwach gefärbten, mit blasenförmigen Kernen versehenen Zellen bestehen. Sie haben also das charakteristische Aussehen der gewöhnlich als Epitheloidzellen in einem Tuberkel benannten Gebilde. In einigen Knötchen kommen auch in den blossen Centren kleinere oder grössere Riesenzellen mit Randstellung der Kerne vor. Die Zellen in dem Infiltrationswall um die blassen Centren berum treten durch Unn a's Methode mit polychromem Methylenblau auf eine besondere Weise hervor. Einen so gefärbten Schnitt habe ich in Fig. 1 gezeichnet. Er stellt einen Theil von einem solchen Knötchen mit dem blassen Centrum nach oben (Fig. 1 a) und dem Infiltrationswall nach unten (Fig. 1 b) dar. Dieser Wall besteht zum grössten Theil aus ziemlich grossen, stark blaugefärbten Zellen (Fig. $1 U$ und ähnliche), die ein stark gefärbtes Protoplasma zeigen, in welchem ein oder żwei grosse hellere Flecke zu sehen sind. Oft sieht man in der Mitte des hellen Fleckes einen dunklen Punkt, der 
deutlich zeigt, dass wir es mit einem Kern und seinen Kernkörperchen zu thun haben. Sehr oft fehlt zwar ein solches Kernkörperchen, aber die ziemlich grosse Aehnlichkeit mit den hellen Flecken, die einen dunklen Punkt in ihrer Mitte haben, macht doch, dass man bei der ersten Betrachtung auch diese hellen Flecke für schwach gefärbte, blasenförmige Zellkerne hält (Fig. $1 \mathrm{UK}$ und ähnliche Lellen). In den Zellen, die eine grössere Protoplasmamenge haben, liegt gewöhnlich der helle Kernfleck mehr oder weniger excentrisch (z. B. die mit $U e$ bezeichneten Zellen in Fig. 1). In den Zellen aber, wo die Grösse des einen oder der beiden hellen Flecke nur einen schmalen Streifen eines Protoplasmasaums übrig lässt, kann man keine excentrische Kernlage beobachten.

Die äussere Form dieser Zellen sind theils eine mehr langgestreckte, theils ungefähr gleich breite und lange, doch, wenn sie sich auch der runden Form nähern, gewöhnlich eine mehr oder weniger deutlich polygonal eckige. Viereckige Formen sind ziemlich gewöhnlich, dreieckige kommen auch vor. Ab und zu besonders in dem äusseren Rand des Infiltrationswalles gegen die Grenze des umgebenden Bindegewebes zu kommen auch solche Zellen von Spindelform vor von verschiedener Grösse. Einige von diesen haben in einem oder beiden Spitzen kürzere oder längere Ausläufer und gehen dadurch zu einem vollkommenen Bindegewebszelltypus über. Man sieht also (Fig. 1 und Fig. $2 Z$ ) besonders nach aussen gegen das umgebende Bindegewebe zu alle möglichen Zwischenformen zwischen den grossen blauen Zellen und typischen Bindegewebszellen.

Ueber die feinere Structur des Protoplasmas dieser Zellen ist schwer etwas bestimmtes zu sagen. Sehr oft kann man gar keine Structur erkennen. Aber in einigen Zellen sieht man deutlich distincte Körner, die am Rande zwischen dem hellen Fleck und dem dunklen Protoplasma ringförmig liegen (z. B. die mit $U r$ bezeichneten Zellen in Fig. 1). Diese Körner sind aber $k$ e in e Protoplasmakörner. Man kann sich nämlich bei genauer Untersuchung überzeugen, dass sie einen Ring von randgestellten feinen Chromatinkörnchen bilden und dem Kern zugehören. Wahrscheinlich kommt ein solcher Kern mit einem Ring von randgestellten Chromatinkörnchen in den meisten von diesen 
Zellen vor. Der Rand des Kernes und die Randkörnchen sind nur durch die starke Protoplasmafärbung mehr oder weniger verdeckt worden. Andere Mal sieht man auch in dem übrigen Protoplasma eine Andeutung zur Körnelung, wie die Zellen $U$ in Fig. 2 zeigen.

Die Farbstärke dieser Zellen bei Färbung mit polychromem Methylenblau ist selbstverständlich etwas verschieden, wenn man mehr oder weniger entfärbt. Aber stets ist doch der Kern schwächer, das Protoplasma stärker gefärbt.

Neben und zwischen diesen grossen blauen Zellen sieht man auch in einigen Knötchen, und wie Fig. $2 T$ zeigt, sogar in sehr grosser Menge, viel kleinere runde doch mehr oder weniger deutlich hervortretend eckige, blassblaue Zellen, die theilweise von einem stärker gefärbten Saum umgeben sind. Auch Zwischenformen zwischen diesen Zellen und Bindegewebszellen sind zu finden. Unveränderte Bindegewebszellen liegen auch zwischen diesen Zellen so wie zwischen den Plasmazellen und scheinen in die Epitheloidzellen des Centrums zu übergehen.

Dazu haben wir noch die sogenannten Mastzellen zu erwähnen, die mit polychromem Methylenblau und mit Kresylviolett gefärbt ein rothes, also metachromatisch gefärbtes, stark körniges Protoplasma zeigen, besonders schön bei der letzteren Färbung. Bei Färbung mit polychromem Methylenblau zeigen die Mastzellen einen Kern, der sehr ähnlich dem Kern der grossen blauen Zellen ist, also schwach blaugefärbt und blasenförmig (Fig. $1 \mathrm{M}$ ). Die Form und Grösse der Mastzellen ist derjenigen der grossen blauen Zellen manchmal sehr ähnlich, Andere Mal sind die Mastzellen mehr lang gestreckt, ja sogar sehr lange schmale und gebogene Formen sind nicht so selten. In einigen Mastzellen ist die Grenze des Protoplasmas gegen aussen nicht scharf, sondern wie zerbrochen, so als ob die Granula aus der Zelle herausfallen wollen.

Die Mastzellen kommen in diesem Präparate in dem Infiltrationswalle der Knötchen zwischen den grossen und kleinen blauen Zellen ziemlich zahlreich vor (Fig. 1 und Fig. $2 M$ ). Bedeutend zahlreicher sind sie aber in dem Bindegewebe zwischen den Knötchen, ja sie kommen sogar hier in ungeheurer Menge oft als lange und gebogene, den Bindegewebszellen 
ähnliche Formen vor. Diese Formen habe ich nicht in dem Infiltrationswalle beobachtet. Hin und wieder findet man auch frei im Gewebe liegende Ansammlungen von rothen Mastzellengranula (Fig. $1 f$ ).

In dem Bindegewebe zwischen den Lupusknötchen trifft man überall mehr Spindelzellen als normal, so dass man auf einen Proliferationsprocess in den Bindegewebszellen schliessen muss. Daneben sieht man kleinere oder grössere Ansammlungen von solchen grossen tiefblauen Zellen, die in dem Infiltrationswalle vorkommen theils in unregelmässigen Herden, theils in breiten oder schmäleren Zügen. Einige davon ragen tief in die Fettgewebssepten herunter. Auch in den kleinen Septen zwischen den verschiedenen Fettzellen sind mehr Bindegewebszellen als normal vorhanden. Ebenso sind hier einige solche grosse stark tingirte Zellen, wie sie in dem Infiltrationswalle vorkommen und Zwischenformen zwischen diesen Zellen und Bindegewebszellen zu finden.

Die kleinen Knötchen, die tief unten vorkommen, bestehen zum grössten Theil aus solchen Epitheloidzellen, welche die blassen Centren in den anderen Knötchen bilden. Dazu sieht man einige Lymphocyten zwischen diesen. In einigen kann man kaum von einem Infiltrationswall sprechen. Da, wo ein solcher vorkommt, ist er so gebaut wie die übrigen, nur bedeutend schwächer ausgebildet.

In den Gefässen dieses Präparates sieht man gewöhnlich zahlreiche rothe Blutkörperchen und polynucleäre Leukocyten. Seltener, aber doch überall in den Gefässen zu sehen, sind eosinophile körnige Zellen. Ziemlich sparsam kommen Lymphocyten vor. Fon diesen Blutzellen sieht man (abgesehen von einer kleinen Blutung auf einer Stelle) im Gewebe nur sehr vereinzelte polynucleäre Leukocyten und Lymphocyten, die letzteren besonders, wie erwähnt, in den kleinsten Knötchen. Eosinophile Zellen habe ich nicht im Gewebe gefunden.

Die grossen tiefblau gefärbten Zellen, von welchen oben schon gesprochen ist, stimmen in allen ihren Eigenschaften mit den Zellen überein, die Unna 1891 in seiner Arbeit „Ueber Plasmazellen insbesondere beim Lupus" (18) beschrieben und Plasmazellen genannt hat. Um diese Uebereinstimmung deut- 
lich zu zeigen, erlaube ich mir zu citiren, wie Unna sie pag. 302 und 303 in seiner Arbeit beschreibt.

„Diese Zellen sind ungemein gross und tief dunkelgefärbt, so dass sie schon bei schwacher Vergrösserung den Eindruck von gewöhnlichen Mastzellen machen. Bei starker Vergrösserung sind sie gekörnt, von nahezu kubischer oder rhombischer Form und den dicht gehäuften Mastzellen bei Urticaria pigmentosa sehr ähnlich. Nur fällt es auf, dass keine langgestreckte Zellformen wie ja stets bei Mastzellen vorkommen, dass keine so regelmässige Anordnung der Form der Körner besteht, dass dieselbe überhaupt viel feiner und statt metachromatisch blauroth oder violett, rein blau gefärbt sind."

„Auf der anderen Seite findet man alle nur wünschenswerthen Uebergänge dieser grossen Zellen nach aussen gegen das gesunde Gewebe hin zu abnorm protoplasmareich und schliesslich zu normalen Bindegewebszellen. ${ }^{\text {" }}$

${ }_{n}$ Aus diesen entstehen also die tiefblauen grossen Zellen durch Zunahme und Abrundung des Zelleibes, während der Kern ebenso gross bläschenförmig und hell bleibt und einen ähnlichen tinctoriellen Gegensatz bei dieser Färbung zum Protoplasma zeigt, wie der Kern der Mastzellen bei ihrer gewöhnlichen Darstellung nur in schwächerem Grade. Er bleibt nämlich in der Färbung zurück und erscheint dadurch als eine hellere Lücke in dem Protoplasma, die sich als Kern oft nur durch das stark tingirte Kernkörperchen documentirt. Die Körnung des Protoplasmas ist so fein, dass selbst die allerstärkste Vergrösserung an isolirt liegende Zellen die einzelnen Körner nicht gut als solche zu erkennen erlauben, also eine Körnerung höherer Ordnung als die der Mastzellen und der Granula von Altmann."

Wenn man weiter meine Abbildungen 1 und 2 (die mit $U$ bezeichnete Zellen und ähnliche) mit den Plasmazellen in Fig. 1 in Unna's Lehrbuch der Histopathologie der Haut vergleicht, so sieht man, dass die Plasmazellen hier mit den grossen blauen Zellen in meinen Präparaten in allen Hauptsachen übereinstimmen. Ich kann also nicht daran zweifeln, dass meine Zellen von derselben Art sind wie Unna's Plasmazellen und nenne darum auch diese Zellen Unna'sche Plasmazellen.

Die kleinen blauen Zellen, die besonders in Fig. $2 T$ vorkommen, kann ich mir nur als Plasmatochterzellen, wie sie Unna genannt hat, erklären.

Durch die verschiedene Färbungsmethoden, die ich gebraucht habe, kann man einige Eigenschaften dieser Plasmazellen kennen lernen. Färbt man z. B. mit einer Kernfarbe wie 
Hämatoxylin, Saffranin, Methylgrün oder Kresylviolett, so sehen die Unna'sche Plasmazellen bei dieser Färbung ganz anders aus als bei Färbung mit polychromem Methylenblau. Man sieht nämlich da einen distinct gefärbten Kern mit theils randgestellten Chromatinkörnchen, theils einem Kernkörperchen in der Mitte und das Protoplasma sehr schwach, aber doch erkennbar gefärbt. Auch dieses stimmt mit Unna's Anschauungen über Plasmazellen überein, denn er sagt in seiner 1892 erschienenen Arbeit "Ueber die Bedeutung der Plasmazellen" (19) über diese Zellen: „Der Kern ist gewöhnlich oval, liegt häufig an einem Ende der Zelle, erscheint bei der Protoplasmatärbung als heller Fleck in der dunkelblauen Zelle, bei Kernfärbung aber zeigt er ein grossbalkiges Chromatinnetz mit einer Reihe sehr grosser, stark tingibler Chromatinkörner oder bei stärkerer Entfärbung nur die letzteren."

Auch mit reinem Methylenblau bekommt man andere Bilder als mit polychromem Methylenblau. Dies ist auch nicht wunderbar, da die beiden Färbungsmethoden eigentlich ganz verschieden sind. Das polychrome Methylenblau, das ich angewendet habe (von E. Leitz in Berlin), enthält gar kein Methylenblau, sondern nur Umwandlungsproducte davon. Das kann man, wie ich von Dr. Leonor Michaëlis in Berlin gehört habe, durch eine einfache Untersuchung bestimmen. Wenn man nämlich zu einer verdünnten Lösung von Methylenblan einige Tropfen Natronlauge setzt, so bleibt nur das reine Methylenblau blau. Die Umsetzungsproducte desselben aber gehen in Roth über, welche Farbe durch Chloroform ausgeschüttelt werden kann. Mein polychromes Methylenblau geht bei dieser Probe vollkommen in Roth über, und nach Ausschütteln mit Chloroform ist keine Spur von blauer Farbe in der Wasserlösung zurïck. Mein reines Methylenblau (aus den Höchster Farbwerken) dagegen bleibt bei Zusatz von Natronlauge blau und bei Ausschütteln mit Chloroform erhält dieses nur eine schwache Spur von rother Farbe.

Eine Probe, ob das Methylenblau rein ist, ist anch dessen Färbung von Mastzellengranula. Nur wenn diese rein blau gefärbt werden, ist das Methylenblau rein. 
Die beiden Farben polychromes und reines Methylenblau sind also ganz verschiedene Farben und so ist es auch begreiflich, dass man verschiedene Resultate durch die beiden Farben erhält.

Wenn man nun Unna's Plasmazellen mit reinem Methylenblau färbt, so bekommt man ein Bild, ziemlich ähnlich als wenn man mit Kernfärbungen färbt. Das Protoplasma ist nur ein wenig stärker gefärbt und die Chromatinkörner nicht so stark, aber man hat doch eine deutliche Kernfärbung.

Wenn dann Schnitte, die mit Kernfärbungen und mit reinem Methylenblau gefärbt sind, mit den durch polychromes Methylenblau gefärbten verglichen werden, so bemerkt man sofort, dass bei der ersten Färbung man viel öfter eine excentrische Kernlage, viel seltener dagegen zwei Kerne sieht als bei Färbung mit polychromem Methylenbau (vergleiche Fig. $1 U$ und ähnliche Zellen, mit den Zellen in Fig. 3). Die mit reinem Methylenblau gefärbten Schnitte zeigen ausserdem als Eigenthümlichkeit zur Seite des excentrisch gelegenen Kerns mehr oder weniger in der Mitte der Zelle eine Aufhellung des Protoplasmas, einen sogenannten Lichthof, der aber nicht immer sehr deutlich ist (siehe Fig. 3).

Durch ein genaues vergleichendes Studium kann man sich überzeugen, dass ein solcher gut ausgeprägter Lichthof bei Färbung mit polychromem Methylenblau eben so wie der Kern in der Färbung zurückbleibt und darum auch als eine runde, helle Lücke in dem tiefblauen Protoplasma sich zeigt, natürlich ohne Kernkörperchen in der Mitte. Wenigstens ein Theil von diesen runden, kernähnlichen, hellen Flecken ohne Kernkörperchen, die bei Färbung mit polychromem Methylenblau hervortreten, stellt also nicht einen Kern, sondern einen Lichth of im Protoplasma dar. Dadurch ist auch erklärt, warum man bei Färbung mit polychromem Methylenblau viel häufiger als bei Kernfärbungen zwei Kerne zu sehen glaubt, da nämlich auch der Lichthof bei dieser Färbung als Kern leicht imponirt.

Auch der andere Unterschied, die öfter vorkommende excentrische Kernlage ist hierdurch erklärt. Denn da der Lichthof als Kern imponirt und in der Mitte der Zelle liegt, kann 
man die in Wirklichkeit vorkommende excentrische Kernlage leicht übersehen. In einigen Fällen trifft man in einer Plasmazelle nur einen hellen Fleck ohne Kernkörperchen. Dieser könnte also entweder ein Kern sein, dessen Kernkörperchen schwächer entwickelt wäre, so dass es sich nicht färbt, oder ein Lichthof. Der Kern müsste im letzteren Fall auf irgend eine Weise versteckt sein. Er kann z. B. tiefer oder höher als der Lichthof liegen und bei einer anderen Einstellung der Mikrometerschraube hervortreten. Ein solcher Fall ist die Zelle $x$ in Fig. 3, die in der Zeichnung nur einen Lichthof zeigt. Dieselbe Zelle ist in Fig. $3 y$ bei höherer Einstellung gezeichnet und zeigt. einen deutlichen Kern auf derselben Stelle, an welcher früher sich ein Lichthof befand.

Was die Färbung mit Thionin betrifft, so habe ich mit dieser Färbung Bilder bekommen, die etwa in der Mitte zwischen polychromem und reinem Methylenblau stehen. Da doch Thionin dem Methylenblau nahe verwandt ist, so ist es auch möglich, dass dasselbe etwas verschieden färben kann, wenn es rein oder zersetzt ist. Die Bilder, die ich bekornmen habe, sind interessant, insofern sie durch ihr Mittelstadium den Vergleich zwischen den mit polychromem und mit reinem Methylenblau gefärbten Schnitten leichter machen. Sie zeigen nämlich gewöhnlich das Protoplasma etwas tiefer blau als den Kern gefärbt. Gewöbnlich sieht man aber die randgestellten Chromatinkörner deutlich.

Ich habe also gefunden, dass die Unna'sche Plasmazellen in diesem Präparat sich durch polychromes Methylenbla u auf eine eigenthümliche Weise färben. Da Unna diese Methode als eine specifische für diese Zellen herrorgehoben hat, so stimmen auch in dieser Hinsicht die hier gefundenen Zellen mit den Zellen, die Unna als Plasmazellen beschrieben hat, überein.

Was weiter die Entstehung der Plasmazellen betrifft, so hat Unn a diese Zellen für veränderte Bindegewebszellen und nicht für Leukocyten gehalten. $\mathrm{Zu}$ derselben Ueberzeugung, dass es sich in diesem Fall um veränderte Bindegewebszellen handelt, bin ich auch durch mein Studium gekommen. Die Gründe hierfür will ich jetzt zusammenfassen: 
1. Ist der Kern dieser Zellen nicht dem Leukocytenkern, sondern dem Bindegewebskern ähnlich. Denn wenn man Leukocyten mit polychromem Methylenblau färbt, so zeigt der Kern immer ein gut entwickeltes Chromatinnetz und nie ein solches blasenförmiges Aussehen, welches viel mehr mit dem Bindegewebskern übereinstimmt.

2. Sind die Zellenformen auch wo sie frei liegen, nicht so rund wie immer bei freien Leukocyten, sondern mehr oder weniger deutlich eckige (drei-, vier-, fünf- oder mehreckige). Manchmal sieht man eine von den Ecken in einen Ausläufer iibergeben, was wiederum die grösste Aehnlichkeit mit Bindegewebszellen aufweist.

3. Sieht man überall, besonders aber an der äusseren Peripherie der Lupusknötchen, alle möglichen Zwischenformen zwischen den Unna'schen Plasmazellen und den Bindegewebszellen (siehe Fig. 1 und 2, $Z$ ).

Hier muss ich die Bemerkung einschalten, dass, wenn man Zwischenformen zwischen zwei verschiedene Zellarten sieht, man annehmen kann, dass entweder die eine Zellform in die andere übergeht oder umgekehrt. Es wandeln sich also bier entweder Bindegewebszellen in Plasmazellen oder Plasmazellen in Bindegewebszellen um. Das sind die beiden einzigen Möglichkeiten, die man sich vorstellen muss, um die Zwischenformen zu erklären. Um darum in diesem Fall zu bestimmen, welche ron diesen beiden Möglichkeiten hier stattfindet, habe ich besonders darauf geachtet, wo man diese Zwischenformen findet. Es ist ja klar, dass die Lupusknötchen im Anfang kleiner sind und dann durch peripherisches Fortschreiten wachsen, so dass die in der Peripherie liegenden Plasmazellen jünger sind als die mehr centralen Plasmazellen. Da wir also sehen, dass die jüngeren Plasma-

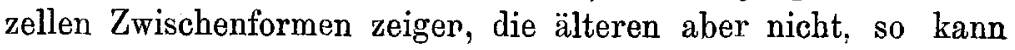
meiner Ansicht nach dies nicht anders gedeutet werden, als dass die Bindegewebszellen sich in Plasmazellen umwandeln.

4. Ausserdem ist in diesem Präparate auffallend, wie gross überall die Bindegewebsproliferation und wie untergeordnet das Vorkommen von Leukocyten im Gewebe ist. Ueberall im Bindegewebe sieht man auch solche Zwischenformen wie in der Peripherie der Knötchen. 
Ich kann also keinen einzigen Grund finden, um die Unna'schen Plasmazellen hier in diesem Präparat als Leukocyten anzusehen. Im Gegentheil sprechen alle Gründe dafür, sie als veränderte Bindegewebszellen zu betrachten.

Da die Mastzellen, die in diesem Präparat in ungeheurer Zahl vorkommen, auch, soviel man jetzt weiss, veränderte Bindegewebszellen sind, so weist also alles darauf hin, dass die Aetiologie des Lupus hier in diesem Fall eine Proliferation und Veränderung des Bindegewebes, weniger dagegen eine Leukocytose and Veränderung von Leukocyten hervorgerufen hat.

Ich gehe jetzt zu einem anderen Fall von Lupus über, der wesentlich verschiedene histologische Veränderungen zeigt und stelle darum diesen Fall dem ersten gegenüber.

Fall2. Lupus vulgaris faciei. Das Präparat besteht aus einem ziemlich grossen Knoten (ungefähr $7 \mathrm{Mm}$. in Durchmesser), dessen Epidermis theils normal, theils etwas hyperplastisch, theils atrophisch mit abgeflachten Papi]len ist. Uebrigens besteht der Knoten aus einem Granulationsgewebe, welches unmittelbar unter der Epidermis nur an einigen runden Stellen dicht, an anderen aber recht dünn ist. Weiter ab von der Epidermis ist das Granulationsgewebe überall dicht und ziemlich, diffus ausgebreitet. Dieses Gewebe ist von grösseren und kleineren Gefässen reichlich durchgezogen, die alle von rothen Blutkörperchen angefüllt sind. (Fig. 4 r.) Dazu enthalten sie zahlreiche polynucleäre Leukocyten und auch Lymphocyten ziemlich reichlich. (Fig. $4 p$ und $l$.) Eosinophile Zellen habe ich trotz grösster Mühe nur einige gefunden. Daneben muss man konstatiren, dass in den meisten Gefässen die Endothelzellenkerne vermehrt und aufgebläht sind, so dass sie in das Lumen der Gefässe hineinwuchern. (Fig. 4 e.)

Uebrigens sieht man in dem Granulationsgewebe theils blasenförmige Bindegewebskerne (Fig. 4 b), die den geschwollenen Endothelzellenkernen sehr ähnlich sind, theils kleine runde Kerne, die den Lymphocytenkernen in den Gefässen gleich sind (Fig. $4 l$ ), theils sparsame polynucleäre Leukocyten (Fig. 4 p). Eosinophile Zellen wurden nicht gefunden. In den mit polychromem Methylenblau gefärbten Sohnitten kommen auch Mastzellen vor, doch ziemlich sparsam, gar nicht im Vergleich mit ihrer Anzahl in dem ersten Fall. Sie sind den Mastzellen in Fall 1 vollkommen ähnlich und haben wie da einen blasenförmigen, den umgebenden Bindegewebskernen sehr ähnlichen Kern.

Sehr zahlreich in diesem Granulationsgewebe sieht man auch grosse, protoplasmareiche Zellen gewöhnlich mit abgerundeten, nicht eckigen Contouren, von ovaler, eiförmiger oder 
cirkelförmiger Form und mit dem Kern immer excentrisch gelegen. Dieser ist in Verhältniss zu dem blassblauen Protoplasma immer sehr stark gefärbt und zeigt eine charakteristische Randstellung von 5-8 grossen Chromatinkörnern, manchmal auch in der Mitte des Kernes ein Chromatinkorn. Das blassblaue Protoplasma zeigt keine deutliche Körnelung, sondern ist beinahe homogen und sehr oft viel stärker in seinen peripheren Theilen gefärbt als in den centralen, wodurch ein heller sogenannter Lichthof central und zur Seite des Kernes im Protoplasma entsteht (Fig. 4 P.). Diese Zellen sind theils sparsam in dem Gewebe, theils sind sie, besonders in der Nähe von Gefässen, sehr zahlreich.

Wie aus dieser Beschreibung herrorgeht, zeigen diese grossen Zellen keine Aehnlichkeit mit den Unna'schen Plasmazellen. Die meisten morphologischen Merkmale dieser Zellen stimmen dagegen überein mit den Zellen, die v. Marschalko in seiner Arbeit "Ueber die sogenannten Plasmazellen, ein Beitrag zur Kenntniss der Herkunft der entzündlichen Infiltrationszellen" (11) als Plasmazellen beschrieben hat. Nur in einer Hinsicht weichen sie v. Marschalko's Beschreibung ab, nämlich dass ihr Protoplasma nicht krümelig, zusammengeballt oder wie zerfetzt, sowie v. Marschalko es beschreibt, sondern beinahe homogen ist. Doch scheint es mir, dass $v$. Marschalko kein grosses Gewicht darauf legen kann, denn die Plasmazellen, die er als Beispiele abbildet, haben auch ein homogenes Protoplasma und wenn man meine Fig. $4(P)$ mit Marschalko's Abbildungen vergleicht, so sieht man deutlich die volle Uebereinstimmung von den Zellen, die er und ich in Fig. $4 P$ abgebildet.

Was übrigens $\nabla$. Marschalko von seinen Plasmazellen sagt, stimmt auch alles mit dem übrigen, was ich in diesen Präparat beobachtet habe. So sagt v. Marschalko, dass es Uebergangsformen von den Lymphocyten zu den Plasmazellen gibt dadurch, dass um einige Lymphocytenkerne ein kleiner Protoplasmasaum gefärbt ist. Dazu kommen andere, die einen etwas grösser gefärbten Saum haben. Je grösser dieser gefärbte Protoplasmasaum ist, desto mehr sind sie seinen Plasmazellen ähnlich. Auch in meinen Präparaten kommen solche 
Zwischenformen $a b$ und $z u$ vor (die mit $Z$ in Fig. 4 bezeichneten Zellen).

Theils in Folge dieser sogenannten Uebergangsformen, theils in Folge anderer Gründe hält v. Marschalko seine Plasmazellen für veränderte Lymphocyten. Was die grossen Zellen in meinen Präparaten betrifft, so spricht $n i c h t s$ gegen eine solche Annahme, im Gegentheil viel dafür. Denn 1. ist der Kern hier, wie Fig. $4 P$ deutlich zeigt, den blasenförmigen Bindegewebskernen sehr unähnlich, stimmt dagegen mit den kleinen ehromatinreichen Lymphocytenkernen überein, 2. ist die Form der Zellen immer rund oder wenigstens abgerundet, 3. gibt es die schon besprochenen Zwischenformen und 4. kommen keine deutliche Zwischenformen zwischen diesen grossen Zellen und Bindegewebszellen vor. Nur etwas langgestreckte Formen, doch immer ohne Ausläufer mit abgerundeten Enden und mit dem chromatinreichen Kern sind vorhanden.

Weiter behauptet v. Marschalko, dass die Färbung mit polychromem Methylenblau für seine Plasmazellen gar keine charakteristische Färbung ist, sondern dass sie sich bei Färbung mit gewöhnlichem Methylenblau so gut wie mit polychromem Methylenblau färben und dass bei den meisten Färbemethoden doch die Plasmazellen durch ihre morphologischen Merkmale sehr gut erkannt werden können. Wie rerhalten sich jetzt die Zellen, die ich in den Präparaten von meinem Fall 2 gefunden habe? In mit polychromem Methylenblau, mit gewöhnlichem Methylenblau und mit Thionin gefärbten Schnitten kann ich keinen Unterschied finden. In den mit Hämatoxylin und anderen Kernfärbungen gefärbten Schnitten ist der einzige Unterschied, dass das Protoplasma nur schwächer gefärbt ist (Fig. 5 P). Im Gegensatz zu den Unna'schen Plasmazellen, die sich mit polychromem Methylenblau auf eine ganz andere und charakteristisch eigenthümliche Weise färben als mit Kernfärbungen und gewöhnlichem Methylenblau, sehen also die Marschalko'schen Plasmazellen bei verschiedenen Färbungen ziemlich ähnlich aus.

Kein Wunder also, dass Unna sagt, dass Plasmazellen mit polychromem Methylenblau in einer specifischen Weise ge - 
färbt werden, v. Marschalko dagegen, dass polychromes Methylenblau keine specifische Färbung für Plasmazellen ist. Jede Behauptung gilt für die besondere Zellenart.

Wir haben also hier in Fall 2 ein Lupusgewebe kennen gelernt von ganz anderer Zusammensetzung als in Fall 1. Im Gegensatz zu diesem haben wir hier wenig Bindegewebsproliferation, auch wenige Mastzellen, aber eine deutlich nachweisbare Leukocytose, und im Gegensatz zu der Veränderung von Bindegewebszellen zu Unna'schen Plasmazellen in Fall 1 haben wir hier eine Umbildung von Leukocyten zu Marschalko's Plasmazellen.

Der Umstand, dass im Lupus so stark einander entgegengesetzte histologische Veränderungen wie in Fall 1 und Fall 2 vorkommen, ist nicht so besonders auffallend. Denn da wir aus der allgemeinen Pathologie wissen, dass das tuberculöse Virus theils Epitheloidzellentuberkel und theils Rundzellentuberikel hervorrufen kann, von welchen der erste mehr Bindegewebsveränderungen, der zweite mehr Leukocytenveränderungen darstellt, ist es ja auch nicht merkwürdig, dass der Tuberkelbacillus, das Virus des Lupus, einmal mehr Bindegewebsveränderungen, ein anderes Mal mehr Leukocytenveränderungen hervorrufen kann.

In den beiden soeben beschriebenen Lupusfällen haben wir also zwei morphologisch und tinctoriell verschiedenartige Zellarten kennen gelernt, die von zwei ver schiedenen Forschern beide Plasmazellen genannt sind, v. Marschalko hat zwar den Namen „Krümelzellen" vorgeschlagen, aber nicht einmal selbst gebraucht. Da in seinen Abbildungen ausserdem das Protoplasma gar nicht krümelig ist, will ich auch diesen Namen nicht brauchen, sondern weiterhin nur von Unna's und Marschalko's Plasmazellen sprechen.

So weit scheint mir alles klar zu sein. Merkwürdigerweise sagt v. Marschalko aber in seiner Arbeit, dass seine Plasmazellen dieselbe Zellart sind wie die von Unna in seiner ersten Arbeit beschriebenen. Es scheint mir aber doch, als liesse sich diese Differenz trotzdem erklären. Wir haben ja gesehen, dass die Unna'schen Plasmazellen, mit reinem Methylenblau gefärbt, ein ganz anderes Aussehen haben als mit polychromem Methylen- 
blau. Und in der That sind sie bei der ersten Färbung viel schwerer von Marschalko's Plasmazellen zu trennen. Denn die stärkere Kernfärbung, die schwächere Protoplasmafärbung, der bei dieser Färbung vom Kern deutlich zu trennende protoplasmatische Lichthof, alles dieses sind Momente, die eine viel grössere Aehnlichkeit mit Marschalko's Zellen aufweisen. Wenn man die Zellen in Fig. 3 mit den Zellen $P$ in Fig. 4 und 5 vergleicht, so sieht man, dass der Unterschied dieser beiden Zellarten nur in der etwas mehr eckigen Zellform mit einer Andeutung zu Ausläufern und in dem etwas zarteren Chromatinnetz der Kerne besteht. Daher können die beiden Zellarten ziemlich leicht bei dieser Färbung mit einander verwechselt werden. Da nun v. Marschalko hauptsächlich mit gewöhnlichem (nicht polychromem) Methylenblau gearbeitet hat, ist es ja sehr begreiflich, dass er die beiden Zellarten nicht von einander trennen konnte, sondern nur eine Art von Plasmazellen sah und da sie nicht Unna's Plasmazellenbeschreibung vollkommen ähnlich waren, so musste er seine neuen morphologischen Merkmale aufstellen. Ich kann wenigstens keine andere Erklärung finden.

Hervorheben muss ich also, dass bis jetzt die beiden Plasmazellarten nurbei Färbung mit polychromem Methylenblan leicht und sicher von einander zu trennen sind. Und da Unna in seinen Arbeiten immer betont, dass seine Färbungsmethode mit polychromem Methylenblau auf einer charakteristischen und specifischen Weise die Plasmazellen färbt, kann ich darin nur einen neuen Beweis sehen für die Identität der Plasmazellen in meinem ersten Präparat mit den Zellen, die Unna in seiner ersten Arbeit als Plasmazellen bezeichnet hat.

Ich bin auch nicht der Erste, der beobachtet hat, dass Unna's und'Marschalko's Beschreibungen verschieden sind und auf zwei Zellarten hinweisen. Schon Unna protestirt in seiner Arbeit: „Ueber Plasmazellen, Antikritisches und Methodologisches" (21) gegen die v. Marschalko aufgestellten morphologischen Merkmale und will nicht alle diese als charakteristisch anerkennen. 
Weiter sagt Krompecher in seinen „Beiträge zur Lehre von den Plasmazellen" (9) (pag. 164) Folgendes: "Doch sowohl bezüglich der Beschaffenheit des Plasmas als auch der des Kernes differiren die Beschreibungen Unna's und v. Mar$\mathrm{schalko's} \mathrm{bedeutend."} \mathrm{Durch} \mathrm{seine} \mathrm{Untersuchungen} \mathrm{ist} \mathrm{Krom}$ pech e $\mathbf{r}$ ebenfalls dahin gekommen, zwei Arten von Plasmazellen aufzustellen. Die eine Art nennt er normale Plasmazellen und von diesen sagt er (pag. 170): „Bezüglich der morphologischen Eigenschaften der typischen Plasmazellen stimmen meine Untersuchungen vollkommen mit denjenigen $v$. Marschalko's überein." "Auch bezüglich der Lagerung und Abstammung der Plasmazellen von Lymphocyten muss ich mich ganz der Anschauung v. Marschalko's anschliessen." Die andere Art nennt er pathologisch veränderte Plasmazellen und beschreibt diese (pag. 172) auf folgende Weise: „Die eigentliche sofort in die Augen springende Abweichung, welche diese pathologischen Plasmazellen auf den ersten Blick von den normalen Plasmazellen unterscheiden lässt, betrifft den Kern." „Der bläschenförmige helle Kern und das zerfetzte, schollige Protoplasma zeichnen daher diese Plasmazellen aus und lassensie sofort vonden normalen Plasmazellen mit dunklem Kern und mehr hellem Protoplasma unterscheiden. Möglicherweise beziehen sich die schon gegebenen Beschreibungen Unna's auf derartige Zellen. Dass Unna solche Zellen gesehen, dafür spricht die Zeichnung von Plasmazellen bei Framboesie, welche Unna in seiner "Histopathologie der Hautkrankheiten" abgebildet und welche unseren soeben beschriebenen pathologischen Plasmazellen zu entsprechen scheinen."

Theils durch die Beschreibung, theils dadurch, dass auch diese Zellen ähnlich sind mit den Zellen, die Unna abbildet, schliesse ich, dass Krompecher unter seinen pathologisch veränderten Plasmazellen dieselbe Zellart versteht, wie ich in meinem ersten Fall beschrieben habe. Auch Krompecher's Abbildungen sprechen dufür.

Was die Herstammung dieser Zellen betrifft, nimmt K ro mpecher an, dass sie nur veränderte Formen der normalen Plasmazellen sind, also derjenigen Zellen, die mit denen Mar- 
schalko's übereinstimmen. Doch spricht er dieses nicht sicher aus, sondern betont nur, dass diese Zellen eine Gruppe für sich bilden. Er sagt nämlich (pag. 174): „Wie immer auch diese Zellen entstehen, soviel steht fest, dass sie eine ges onderte, gutcharakterisirte Gruppeder Plasmazellen bilden und als solche, wie es scheint, hauptsächlich bei infectiöseu Processen in grosser Menge anzutreffen sind."

Auch Joannovics unterscheidet in seiner Arbeit „Ueber das Vorkommen, die Bedeutung und Herkunft der Unna'schen Plasmazellen" (7) zwei Arten von Plasmazellen, von welchen er die eine Art übereinstimmend mit Marschalko's Zellentyp beschreibt, die andere Art dagegen durch ihren grossen hellen und chromatinarmen Kern charakterisirt und als veränderte Bindegewebszellen betrachtet. Besonders deutlich konnte er diese beide Zellgattungen "in den Präparaten nach 24-stündiger Einwirkung des Terpentins unterscheiden, wo wir die Umwandlung von Lymphocyten und polymorphkernigen Leukocyten in Plasmazellerı der ersten Art geradezu verfolgen konnten, während die zweite aus Bindegewebszellen hervorzugehen scheint" (pag. 184).

Hervorheben muss ich, dass diese beiden Forscher, K ro mpecher und Joannovies, mit polychromem Methylenblau gearbeitet haben. Es stimmt also dies mit meinen früheren Bemerkungen über die Bedeutung der Erkennung von Unnaschen Plasmazellen durch die Färbung mit polychromem Methylenblau überein. Ich glaube also, dass es mit dieser Färbung nicht schwer ist, die beiden verschiedenen Zellarten zu trennen.

In einem Nachtrag zu seiner letzten Arbeit über Plasmazellen (13) hat Marschalko gegen Joannovic's Anschauung protestirt. Er meint, dass Joannovic's zweite Art von Plasmazellen keine Plasmazellen sind, weil sie nicht die morphologischen Merkmale haben die v. Marschalko für Plasmazellen aufgestellt hat. Wahrscheinlich macht er gegen mich auch dieselbe Einwendung, dass meine erst beschriebenen Zellen aus demselben Grund keine Plasmazellen sind. Ich muss aber dem gegenüber betonen, dass hier nur ein Streit um Worte und obne wissenschaftlichen Werth vorliegt. Die Hauptsache 
ist, dass es zwei verschiedene Zellarten gibt. Welche von diesen man Plasmazellen nennt, ist wissenschaftlich ziemlich gleichgültig. Aber wenn man nur eine Art als Plasmazellen bezeichnen will, muss man doch die Art, die erstvon Unna als Plasmazellen beschrieben ist, so bezeichnen und nicht diejenigen, welche Marschalko später charakterisirt hat. Denn Unna hat unzweifelhalft das Recht der Benennung für die von ihm zuerst aufgefundenen Zellen. Man kann aber auch den Namen für beide Arten brauchen, da sie doch einige Aehnlichkeiten mit einander aufweisen.

Ueber die Abstammung der beiden Plasmazellenarten sei hier noch etwas angefügt. Ich habe oben Gründe angegeben, um die zuerst beschriebenen Plasmazellen als Bindegewebszellen, die zweiten als Leukocyten anzusehen. Ich bin also zu demselben Resultate wie Joannovic's gekommen. Krompecher ist dagegen zu dem Resultat gelangt, dass die Unnaschen Plasmazellen nur eine veränderte Form der Mar s chalkoschen Zellen sind. - Ich möchte nur im Anschlusse daran betonen, wie schwer es ist über die Abstammung einer Zellart etwas sicheres zu sagen, weil das morphologische Aussehen zu wenig eine Zellart charakterisirt. Dass die Bindegewebszellen in pathologischen Processen sich morphologisch bedeutend verändern können und sogar Leukocyten ähnlich werden, ist eine bekannte Thatsache. Auch sagt Marschalko selbst (pag. 10): "Morphologisch ist es nicht gelungen die mononucleären Leukocyten zu trennen von den jungen wandernden Bindegewebszellen." In seiner Arbeit: "Die Rolle der fixen Zellen in der Entzündung“ (1) erwähnt Baumgarten, dass von einigen Forschern angenommen wird, dass Rundzellen von Aussehen der grossen und kleinen mononucleären Leukocyten zum theil wenigstens aus wuchernden Bindegewebszellen hervorgehen können. Weiter will ich noch erwähnen, dass F. M a rchand (10) neuerdings bei Untersuchungen am künstlich in Entzündung versetzten Netz von Meerschweinchen Beobachtungen gemacht, aus denen hervorgeht, dass aus wuchernden Adrentitialzellen der kleinen Gefässe eine Brut amöboider Rundzellen sich bilden, welche in keiner Weise von den grosskernigen mononucleären Leukocyten des Blutes zu unterscheiden sind. Durch successive mito- 
tische Theilung dieser Zellen entstehen sodann immer kleinere Zellformen, die schliesslich in nichts von kleinen mononucleären Leukocyten, typischen Lymphocyten, abweichen. Marchand nennt die in Rede stehenden Zellen leukocytoide Zellen und ist geneigt, eine Einwanderung derselben in die Blutgefässe anzunehmen, wonach sie die Rolle echter Blutleukocyten zu übernehmen befähigt sein würden. Marchand's Beobachtungen sind von Borst (2) vollkommen bestätigt worden.

Bei dem jetzigen Standpunkt der Wissenschaft darf man also bei der Unterscheidung von Leukocyten und Bindegewebszellen auf die morphologischen Formen kein zu grosses Gewicht legen.

Wenn meine Beobachtungen und Schlussfolgerungen vollkommen richtig sind und also in den beiden beschriebenen Fällen Unna's Plasmazellen wirklich aus Bindegewebszellen und Marschalko's aus Leukocyten entstanden sind, so ist dadurch doch nur bewiesen, dass Unna's Plasmazellen aus Bindegewebszellen und Marschalko's aus Leukocyten entstehen können. Die andere Möglichkeit, dass diese Zellformen auch aus anderen Gebilden entstehen können, muss man noch ausserdem offen halten. Ueber die Wahrscheinlichkeit oder Unwahrscheinlichkeit dieser Möglichkeit will ich mich nicht aussprechen, sondern nur dieselbe betonen und hervorheben, dass die Un. möglichkeit eines anderen Entstehen desselben Zelltypus noch nicht bewiesen ist.

Ich gehe jetzt zu meinen anderen Fällen über and will da einige erwähnen, die von demselben Typus sind wie die beiden ersten.

Fall 3. Lupus vulgaris der Wange. Epidermis ist hypertrophisch mit vergrösserten und verlängerten Papillen. Im Papillarkörper und in der Cutis ist ein stark vermehrter Zellenreichthum. Die zahlreichen Bindegewebszellen haben blasse, spindelförmige und blasenförmige Kerne gewöhnlich mit einem stark hervortretenden Kernkörperchen. Hier und $\mathrm{da}$ in diesem Gewebe sieht man mehr oder weniger runde Ansammlungen von blassen Epitheloidzellen, oft mit Riesenzellen in der Mitte. Theils in den concentrischen Lagen von Bindegewebszellen, die die Epitheloidzellansammlungen umgeben, theils auch in dem übrigen Bindegewebe kommen grosse, stark gefärbte Zellen vor, entweder mehr vereinzelt oder gruppirt, die sich bei näherer Untersuchung als Un na'sche Plasmazellen erwiesen, also mit polychromem Methylenblau gefärbte, ein tiefblaues Protoplasma und einen blasenförmigen Kern mit einem Kern- 
körperchen in der Mitte, mit reinem Methylenblau aber und mit Kernfärbungen ein mehr hellblaues Protoplasma und einen Kern mit distincten Chromatinkörnchen zeigen.

Zerstreut in dem ganzen Bindegewebe kommen Mastzellen von länglichen Formen vor. Von Leukocyten sieht man in dem Gewebe nur einige kleine Lymphocytenansammlungen. In den spärlichen Gefässen kommen einige polynucleäre Leukocyten und Lymphocyten vor. Eosinophile Zellen habe ich nicht gefunden.

Fall 4. Lupus vulgaris der Zehen. Epidermis hypertrophisch mit vergrösserten und verlängerten Retezapfen. Im Papillarkörper und in der Cutis hie und da Riesenzellen mit Epitheloidzellen um sich herum. Nach aussen von denselben einen mehr oder weniger ausgeprägten Wall, bestehend theils aus Bindegewebszellen mit blassen Kernen, die in die Epitheloidzellen übergehen, theils Plasmazellen von Marschalko's Typus, theils Lymphocyten und auch einige Zwischenformen zwischen diesen und den Plasmazellen. Im Cutisgewebe ist etwas narbiges Bindegewebe vorhanden. An einigen Stellen ist dieses Narbegewebe von Zellsträngen durchzogen, die aus Bindegewebszellen und schönen Marschal • $\mathrm{k} \mathrm{o}$ 'schen Plasmazellen bestehen. Nur vereinzeite Mastzellen sind in dem Präparate za seben. Keine eosinophile Zellen sind zu finden.

Diese beiden Fälle 3 und 4 entsprechen also den beiden ersten Fällen. Fall 3 hat denselben Typus wie Fall 1, nämlich reichliche Bindegewebsproliferation mit zahlreichen Mastzellen auf der einen Seite und wenig ausgeprägte Leukocytosis auf der anderen. Die Plasmazellen haben den Typus von Unnaschen Plasmazellen. Fall 4 hat ähnlichen Typus mit Fall 2, nämlich weniger ausgeprägte Bindegewebsproliferation, spärliche Mastzellen, aber eine deutliche Leukocytose, und die Plasmazellen haben den M arschalko'schen Typus.

So typisch wie in diesen Fällen sind aber nicht immer die Plasmazellen ausgeprägt. In vielen Fällen sind durch degenerative oder andere Processe die Zelltypen nicht so leicht von einander zu halten. Es ist ja klar, dass wenn z. B. degenerative Processe im Protoplasma der U n n a'schen Plasmazellen vorkommen, dieses Protoplasma nicht so stark gefärbt wird und also nicht den Kernrand mit seinen Chromatinkörnchen verbirgt, sondern dass der Kern und seine Lage viel leichter zu sehen sind, als wenn das Protoplasma so wie im Fall 1 gut gefärbt ist. Dadurch, dass auf solcher Weise der Kern leichter zu sehen ist und das Protoplasma nicht so gleichmässig tiefblau ist, kann man leicht gewisse Aehnlichkeiten mit Marschal- 
k o's Plasmazellen finden. Trotzdem gelingt es doch gewöhnlich die beiden Zellarten von einander zu scheiden. Die nächst folgenden Fälle zeigen einige Beispiele davon.

Fall 5. Lupus vulgaris der Wange. Epidermis unverändert. In der Cutis theils gut ansgeprägte Lupusknötchen, so wie in Fall 1 mit blassen Centren und stark gefärbten Infiltrationswall um diese herum, theils nur eine Ansammlung von starkgefärbten Zellen. Im Bindegewebe sieht man bedeutend reichlichere Zellen als normal. Dazu treten grosse tiefblaue Zellen in Gruppen oder Strängen. In den Gefässen, die nicht so zahlreich sind, sieht man eine Vermehrung von Endothelzellen, weiter Lymphocyten und polynucleäre Leukocyten. Im Gewebe findet man diese Zellen sehr sparsam. Eosinophile Zellen sind nicht vorhanden. Dagegen findet man ziemlich zahlreiche Mastzellen überall im Bindegewebe. Diese Zellen haben immer einen Kern, der denjenigen der Bindegewebszellen ähnlich ist.

Das feinere Zellstudium in diesem Fall zeigt, dass der Infiltrationswall so wie in Fall I zum grossen Theil aus grossen blauen Plasmazellen besteht. Von diesen sind nur einige den Unn a'schen Plasmazellen vollkommen ähnlich mit ihrem als hellen Fleck hervortretende Kerne, dessen Kernkörperchen in der Mitte deutlich sich zeigt und das stark gefärbte Protoplasma um diesen herum (solche Zellen sind die Zellen $U$ in Fig. 6 und $i$ ). Die meisten Zellen haben ein zerfetztes oder in Klumpen vertheiltes Protoplasma, das nach aussen oft nicht scharf begrenzt sondern mehr franzenförmig ist. (Fig. 6 und $7 d$ und Fig. 8.) In einigen Zellen kann man auch eine mehr oder weniger deutliche Vacuolenbildung sehen. In Folge dessen ist das Protoplasma schwächer und ungleichmässig gefärbt. Oft sind die einzelnen Klumpen gerade so stark gefärbt wie in den typischen Unna'schen Plasmazellen, aber das Ganze macht doch einen schwächer gefärbten Eindruck und lässt in diesen Zellen den Kern immer deutlicher hervortreten mit den oft schön ausgeprägten randgestellten Chromatinkörnchen, die in Fall 1 nur hin und wieder zu sehen waren. Dass hier das Protoplasma durch degenerative Processe in Zerfall begriffen ist, scheint mir ohne weiteres deutlich zu sein. Der Zerfall scheint oft in der Mitte des Protoplasmas zu beginnen, denn gewöhnlich ist die Färbung central viel schwächer als peripher.

Durch die schwächere Färbung des Protoplasmas und das stärkere Herrortreten des Kernes bekommen diese Zellen eine 
gewisse Aehnlichkeit mit Marschalko's Plasmazellen. Bei einem genaueren Studium findet man aber doch Verschiedenheiten. Erstens hat nämlich der Kern mit polychromem Methylenblau gefärbt keine solchen grossen Chromatinkörner, wie die Lymphocyten und Marschalko's Plasmazellen mit derselben Färbung zeigen. Sie sind den herumliegenden Bindegewebskernen und Mastzellenkernen sehr ähnlich. Mit gewöhnlichem Methylenblau und Kernfärbungen gefürbt haben sie (siehe Fig. 9) nicht dasselbe Aussehen wie Marschalko's Zellen, sondern zeigen zahlreichere und grössere Chromatinkörner.

Weiter ist wohl das Protoplasma als ganzes schwächer gefärbt, aber Theile oder Klumpen von demselben sind doch bei der Färbung mit polychromem Methylenblau so stark wie das Chromatin gefärbt, wenn nicht der Zerfallsprocess zu weit gegangen ist. Es färbt sich mit reinem Methylenblau viel schwächer (vergleiche Fig. 9 mit Fig. 6 und $7 d$ ). Dazu muss ich noch hervorheben, dass, wie man in Fig. 6,7 und 8 oft verfolgen kann. die Zellen durch mehr oder weniger fortgeschrittenen Protoplasmazerfall denselben Typus wie in Fall 1 zu einem ganz anderen Typus verändern. Ich muss auch erwähnen, dass Krompecher in seiner zweiten Art von Plasmazellen, welche er für dieselbe hält, die $U n n$ a beschrieben und abgebildet hat, sehr viel von degenerativen Protoplasmaprocessen spricht und, wie das Citat oben zeigt, hält er sogar ein zerfetztes und scholliges Protoplasma für diese Zellart als charakteristisch.

Wenn man alles dieses berücksichtigt, gibt es wohl keinen Zweifel, dass diese Zellen Unna'sche Plasmazellen sind, in denen ein Protoplasmazerfall stattfindet. Hiermit stimmt auch, dass wir hier überwiegend Bindegewebsproliferation, ziemlich reichliche Mastzellen und weniger ausgeprägte Leukocytose haben.

Ein ähnlicher Fall ist:

Fall 6. Lupus vulgaris faciei. Die Epidermis etwas verdickt, nur auf einigen kleinen Stellen sehr verdünnt. In der Cutis überall eine sehr lebhafte Bindegewebsproliferation. Kerntheilungsfiguren siebt man zuweilen. (Fig. $10 \mathrm{k}$ ) Man sieht massenhaft spindelförmige und ovale Zellen theils mit, theils ohne Ausläufer zwischen den Bindegewebsbündeln sich anhäufen. An einigen Stellen zwischen diesen Bündeln sind grössere 
oder kleinere Ansammlungen von Plasmazellen vorhanden. Tuberkelähnliche Lupusknötchen, so wie in Fall 1, 3 und 5 kommen nicht vor, sondern das Ganze besteht nur ans Bindegewebsproliferation und Plasmazellenanhäufungen. Diese letztgenannten sieht man sehr oft um die Gefässe herum. In den Gefässen sieht man sehr wenige polynucleäre Leukocyten und noch weniger Lymphocyten, dagegen sind die Endothelzellen der Gefässe stark vermehrt und angeschwollen. Auch die Perithelzellen der Gefässe sind vermehrt. Im Gewebe sind Leukocyten selten und nur hie und da kommen Ansammlungen von lymphocytenäbnlichen Zellen vor. Mastzellen dagegen sind zahlreich und baben immer einen bindegewebsähnlichen Kern. Eosinophile Zellen wurden nicht gefunden.

Die Plasmazellen sind hier vollkommen ähnliche mit denjenigen im Fall 5, so dass man theils Formen von typischen Unna'schen Plasmazellen sieht, theils solche, wo durch Protoplasmazerfall der Kern deutlicher hervortritt. Die Farbenreactionen sind die für Unna'sche Plasmazellen gewöhnlichen.

Fall 7. Lupus vulgaris faciei. Mehrere runde gut ausgebildete Ansammlungen von Epitheloidzellen mit einer grossen Riesenzelle in der Mitte und oft mehrere kleine daneben. Sie sind gewöhnlich von einem gut ausgebildeten Infiltrationswalle umgeben, doch so, dass die verschiedenen Ansammlungen von Epitheloidzellen zu zwei Gruppen angeordnet sind. In diesen Wällen sieht man eine reichliche Menge runder, lymphocytenähnlicher Kerne. Zwischen diesen liegen blasenförmige Bindegewebszellkerne, die centralwärts in die Epitheloidzellen überzugehen scheinen. Daneben sieht man auch Plasmazellen gewöhnlich mit einem mehr oder weniger zerfallenen Protoplasma und nicht reichlich chromatinhaltigem Kern. Auch Zwischenformen zwischen diesen Plasmazellen und den Bindegewebszellen sind zu finden.

In dem Gewebe ausserhalb dieser Gebilde ist theils eine reichliche Bindegewebsinfiltration, theils eine Infiltration von lymphocytenähnlichen Zellkernen. Auch ziemlich reicbliche Plasmazellen kommen vor. Mastzellen sind nicht so häufig vorhanden, sie zeigen immer einen Kern, der denjenigen der Plasmazellen ähnlich sind. Polynucleäre Leukocyten sind ebenfalls in dem Gewebe zu finden. Die zahlreichen Gefässe in dem Infiltrationswalle haben vermehrte und angeschwollene Endothelzellenkerne, und im Lumen sieht man polynucleäre Leukocyten und Lymphocyten. Eosinophile Zellen wurden in dem Präparate nicht gefunden.

Die Plasmazellen, die hier vorkommen, sind denen im Fall 5 ziemlich ähnlich. Man findet also hier theils Zellen, deren Protoplasma tiefblau gefärbt ist und nur einen hellen Fleck mit Kernkörperchen als Kern zeigt, und theils solche, wo ein mehr oder weniger fortgeschrittener Zerfall des Protoplasmas, besonders central in der Zelle, den Kern sebr deutlich 
hervortreten lässt. Der Kern, mit polychromem Methylenblau gefärbt, hat ein zarteres und mit Kernfärbungen ein stärkeres Chromatinnetz.

In vielen Fällen sieht man aber sehr wenige oder gar keine Plasmazellen.

Fall 8. Lupus vulgaris der Wange. Das Präparat besteht aus einem ziemlich grossen Hautknoten, ungefähr so wie in Fall 2, dessen Epidermis ziemlich unverändert ist. Das Gewebe in dem Knoten zeigt ein schön ausgeprägtes Mosaik von blassen runden Epitheloidzellenanhänfungen und um diese herum und zwischen denselben bogenförmig und guirlandenartig sich schlingende tiefblaue Infiltrationszonen. Diese bestehen aus kleinen runden lymphocytenähnlichen Zellen and zwischen diesen ovale runde oder längliche, mehr blasenförmige Bindegewebszellenkerne, die am Rande in die Epitheloidzellen überzugehen scheinen. Auch kommen Mastzellen mehr oder weniger reichlich vor. Einige von diesen sind vollkommen runde und haben einen Leukocytenähn lichen Kern. Die Gefässe zeigen sämmtliche vermehrte und angeschwollene Endothelzellenkerne und im Lumen sieht man polynucleäre Leukocyten, Lymphocyten und gar nicht selten schöne eosinophile Zellen. Die polynucleären Leukocyten trifft man nicht im Gewebe ausserhalb der Gefässe, schöne eosinophile Zellen dagegen ziemlich häufig.

Gut ausgeprägte Plasmazellen habe ich nicht gefunden. Nur einige von den runden leukocytenähnlichen Kernen sind von einem kleineren oder grösseren Protoplasmahof umgeben. Vielleicht lag hier also ein Beginn zur Plasmazellenbildung vor.

Fall 9. Lupus der Fingerspitze. Wir sehen hier ein von ziemlich reichlichen Gefässen durchgesetztes Granulationsgewebe. Die Gefässe zeigen in ihren Wänden eine sehr grosse Menge von angeschwollenen Endothelzellenkernen und enthalten reichliche polynu. cleäre Leukocyten und Lymphocyten. Das Gewebe selbst besteht aus zahlreichen Lymphocyten und damit gemischte Bindegewebszellen mit mehr oder weniger blasenförmigen Kernen. Auch sieht man polynucleäre Leukocyten ziemlich zahlreich und Mastzellen von länglichen Formen. Un einige lymphocytenähnliche Kerne sieht man einen kleineren oder grösseren Protoplasmasaum, aber keine gut ausgebildete Plasmazellen.

Fall 10. Lupus vulgaris faciei. Dieser Fall zeigt eine bedeutende Bindegewebsproliferation und eine sehr grosse Menge schöner Mastzellen besonders um die Schweissdrüsen und die spärlichen Blutgefässe herum. Plasmazellen und typische Lymphocyten habe ich nicht entdeckt.

Fall 11. Lupus vulgaris des Oberarmes. Das Präparat besteht aus einem ziemlich grossen Knoten mit etwas verdünntem Epidermis und ist erfüllt von einem diffusen Granulationsgewebe, welches aus Bindegewebszellen und Leukocyten zusammengesetzt ist. An einigen. 
Stellen circumscripte Epitheloidzellansammlung ohne Riesenzellen. Mastzellen kommen sparsum vor. Plasmazellen sind nicht gefunden.

Fall 12. Lupus vulgaris des Handgelenkes. Ein diffuses Infiltrat von gemischten Bindegewebszellen und Leukocyten. Keine eigentliche Epitheloidzellanhäufungen, nur hier und da eine Riesenzelle mit einigen Epitheloidzellen neben sich. Ziemlich reichlich sind Mastzellen, gewöhnlich mit stark gefärbtem Kerne, dagegen keine Plasmazellen zu finden.

Fall 13. Lupus vulgaris faciei. Unter der ziemlich unveränderten Epidermis sieht man um die mit weissen und rothen Blutkörperchen gefüllten Blutgefässe herum eine ziemlich reichliche perivasculäre Bindegewebsproliferation. Im Gewebe zwischen den Gefässen ist mässige Proliferation vorhanden. Hin und wieder sieht man Leukocyten ausserhalb der Gefässe. Mastzellen in langgestreckten Formen findet man nicht so selten, aber keine deutliche Plasmazellen.

Fall 14. Lupus vulgaris der Glutealgegend. Ein gewöhnlich gemischtes Granulationsgewebe ohne etwas besonderes.

Fall 15. Lupus vulgaris faciei. Ungefähr so wie Fall 14.

Ich gehe jetzt zu einigen Fällen von Tuberculosis cutis verrucosa über.

Fall 16. Tuberculosis cutis verrucosa. Die Epidermis ist über 5 papillenartigen Erhebungen verdickt, welche aus einem Granulationsgewebe von nicht dicht liegenden Spindelzellen bestehen. In diesem Gewebe finden sich runde blasse Epitheloidzellenanhäufungen umgeben von einem ausgeprägten Wall von stärker gefärbten Zellen. Diese zeigen sich bei näherer Untersuchung als mehr oder weniger degenerirende Unna'sche Plasmazellen. Mastzellen kommen in den Granulationen ziemlich reichlich vor. Wenige Lymphocyten und polynucleäre Leukocyten sind im Gewebe zu sehen.

Fall 17. Tuberculosis cutis verrucosader Hand. Epidermis bedeutend hypertrophisch mit vergrösserten und verdickten Papillen. Im Corpus papillare und in der Cutis ein Infiltrat, hauptsächlich aus Spindelzellen bestehend. Daneben kommen auch reichliche runde lymphocytenähnliche Zellen und Mastzellen vor. An einigen Stellen auch Epitheloidzellenanhäufungen mit Riesenzellen. Theils neben diesen, theils mehr zerstreut, kommen Plasmazellen vor. Diese zeigen dasselbe Aussehen wie diejenigen im vorigen Fall. Die Gefässe zeigen eine sehr reichliche Vermehrung der Endothelzellenkerne, aber sehr wenige Leukocyten in dem Lumen.

Diese beiden Fälle zeigen also hauptsächlich Bindegewebsveränderungen, reichliche Mastzellen, wenige Leukocytose und mehr oder weniger Unna'sche Plasmazellen, sowie es viele Lupusfälle auch gezeigt haben.

Fall 18. Tuberculosis cutis verrucosa. In der Cutis ein circumscriptes Granulationsgewebe von Spindelzellen und lymphocyten- 
ähnlichen Zellen mit einigen Anhäufungen von Epitheloidzellen. In den anderen Theilen der Cutis vermehrte Spindelzellen und reichliche Mastzellen, aber keine deutliche Plasmazellen zu sehen.

Fall 19. Tuberculosis cutis verrucosa. In der Cutis eine reichliche perivasculäre Bindegewebsproliferation um die von rothen und weissen Blutkörperchen gefüllten Blutgefässe herum. Auch zwischen den Gefässen vermehrte Bindegewebszellen. Mastzellen und lymphocytenähnliche Zellen sind auch zu sehen, aber keine Plasmazellen.

Fall 20. Melktuberculose der rechten Hand. Epidermis bedeutend hypertrophisch mit vergrösserten Papillen. In der Cutis eine Gewebsinfiltration, die zum grossen Theil aus Bindegewebszellen besteht. Ausserdem runde lymphocytenähnliche Kerne und spärliche Plasmazellen, die ziemlich klein und nicht so gut ausgebildet sind, aber doch den Unna'schen Plasmazellen zu entsprechen scheinen. Viele Mastzellen, theils von langgestreckter, theils runder Form. Die Gefässwände zeigen vermehrte Endothelzellen, aber Leukocyten sind in denselben kaum zu sehen.

Fall 21. Tuberculöse Lymphdrüse. Das Präparat stellt ungefähr die eine Hälfte einer kleinen Drüse dar. In der Mitte sieht man einige nekrotische Herde und ausserdem dichtgelegene, gut ausgeprägte den Lupusknötchen sehr ähnliche kleine Tuberkel gewöhnlich mit einer grossen Riesenzelle in der Mitte und einem Infiltrationswali von kleinen gutgefärbten Zellen peripher. In der Peripherie der Drüsen sind diese Tuberkel am besten ausgebildet. Der Infiltrationswall besteht aus zahlreichen Lymphocyten und dazwischen blassen, blasenförmigen Bindegewebskernen, die in die Epitheloidzellen der blassen Centra zu übergehen scheinen. In den Gefässen sparsame polynucleäre und mononucleäre Leukocyten. Keine eosinophile Zellen. Theils in dem Infiltrationswalle, doch gewöhnlich mehr in den centralen als in den peripheren, am deutlichsten entwickelte Tuberkeln, theils auch da, wo keine Tuberkel $z u$ sehen sind, kommen gruppenweise Plasmazellen vor, die mit polychromem Methylenblau gefärbt einen stark dunkelblau, mit groben Chromatinkörnern versehenen Kern und ein mehr hellblaues Protoplasma, oft mit einem perinucleären Lichthof neben dern excentrischen Kern, zeigen. Auch mit reinem Methylenblau und mit Hämatoxylin zeigen diese Plasmazellen denselben Typus. Sie sind also Plasmazellen von Marschalko's Typus.

\section{Zusammenfassung.}

Die Beobachtungen, die ich an diesen 21 untersuchten Fällen gemacht habe, sind etwa folgende:

Die histologische Zusammensetzung des Lupusgewebes ist in verschiedenen Fällen etwas verschieden. Von den beiden Elementen, Bindegewebszellen und Leukocyten, die das Granulationsgewebe hier bilden, uiberwiegt bald das eine, bald das andere mehr als Hauptbestandtheil. Meistens findet sich ein sehr 
charakteristisches Gewebe von runden tuberkelähnlichen Knötchen mit blassen degenerirten Centren und einem stärker gefärbten Reactions- oder Infiltrationswall um dieses rings herum. Andere Mal allerdings findet sich nur ein mehr diffus ausgebreitetes Granulationsgewebe. Diese beiden letzten Formen hat Unna in seinem Lehrbuch sehr deutlich als circumscripte oder noduläre und diffus oder radiäre Form aus einander gehalten.

In diesem Lupusgewebe habe ich in vielen, doch nicht in allen Fällen ziemlich grosse und protoplasmareiche Zellen gefunden, die von zwei verschiedenen Typen sind. Der eine Typus zeigt die ron Unna als Plasmazellen beschriebenen Gebilde, der andere die ron Marschalko unter demselben Namen beschriebenen.

Da, wo diese Zellen gut ausgebildete sind, kann man sie durch ihre morphologischen, hauptsächlich und am leichtesten aber durch ihre tinctoriellen Figenschaften unterscheiden. Bei der Unna'schen Färbungsmethode mit polychromem Methylenblau färben sich Marschalko's Plasmazellen auf dieselbe Weise wie mit den meisten anderen Färbemethoden. Die Unnaschen Plasmazellen aber bekommen ein ganz anderes Aussehen dadurch, dass der Kern sich schwächer, das Protoplasma. aber, wenn es nicht degenerirt ist, sich viel stärker färbt.

In vielen Fällen haben die Unna'schen Plasmazellen in Folge von Zerfall und Degeneration des Protoplasmas die specifische Färbung mit polychromem Methylenblau nicht mehr angenommen. Dann erfordert es grosse Aufmerksamkeit, um diese Zellart ron den Marschalko'schen Plasmazellen zu trennen.

Was die Histologie dieser Zellen übrigens betrifft, so zeigen beide gewöhnlich einige histologische Eigenthümlichkeiten. Bemerkenswerth ist, dass diese in beiden Arten dieselben sind, nämlich eine excentrische Kernlage, ein centraler Lichthof und eine Randstellung der feineren oder grösseren Chromatinkörner des Kernes. Wie oben angegeben ist, treten diese Verhältnisse bei den Unna'schen Plasmazellen bei der Färbung mit polychromem Methylenblau nicht deutlich hervor, wenn das Protoplasma nicht degenerirt ist, sondern am besten bei Färbung mit reinem Methylenblau. 
Bisher habe ich keine Fälle gesehen, wo Unna'sche und M arschalk o'sche Plasmazellen zusammen rorkommen, sondern ich habe nur eine von den beidem Arten in dem betreffenden Falle angetroffen. Die Un na'schen Plasmazellen bekam ich dort zu sehen, wo die Bindegewebsveränderungen überwiegen und zahlreiche Mastzellen vorkommen, Marschalko's dagegen, wo die Leukocytose mehr ausgeprägt war und Bindegewebsveränderungen und Mastzellen spärlicher waren.

In den meisten der untersuchten Fälle habe ich die Unnaschen Plasmazellen getroffen. Es stimmt dies überein mit Krompecher's Beobachtungen, dass seine zweite Art ron Plasmazellen hauptsächlich bei infectiösen Processen vorkomme.

Was die Herstammung der Plasmazellen betrifft, so habe ich in meinen Fällen Gründe gefunden, um die Unna'schen Plasmazellen da als veränderte Bindegewebszellen, die Marschalko'schen als veränderte Leukocyten zu betrachten. Ich habe aber auch betont, dass man aus diesen Beobachtungen in meinen Fällen eigentlich keine allgemein giltige Schlussfolge über die Entstehungsweise dieser Zellen ziehen kann, und die Möglichkeit einer anderen Entstehung dieser Zellen ebenfalls hervorgehoben.

Ueber das weitere Schicksal dieser Plasmazellen habe ich keine Beobachtungen machen können. Nie habe ich beobachtet, dass Plasmazellen in die Epitheloidzellen des Lupusknötchens übergehen. Mir schien es immer, als ob die gewöhnlichen Bindegewebszellen, die zwischen den Plasmazellen lagen, sich zu Epitheloidzellen umbildeten. Da Unna's Plasmazellen auch wahrscheinlich Bindegewebszellen sind, scheint in der That eine solche Umbildung sehr möglich zu sein. Schottländer(16) bezeichnet ebenfalls als sehr wahrscheinlich, dass "durch einen Degenerationsvorgang aus der ursprünglichen Plasmazelle eine Epitheloidzelle wird".

Wie ich in der Einleitung zu meiner Arbeit sagte, finde ich die Plasmazellenfrage gar nicht erklärt. Jedem, der das Wort Plasmazellen hört, drängt sich naturgemäss die Frage auf: "Was sind Plasmazellen? Wenn wir einmal sicher und bestimmt 
antworten können: Plasmazellen sind veränderte Bindegewebszellen oder veränderte Leukocyten oder beides, so ist aber damit nicht die Frage, was Plasmazellen sind, beantwortet sondern nur der erste Theil dieser Frage. Denn wir müssen nämlich auch sagen können, worin diese Veränderungen bestehen, die die Zellen zu Plasmazellen umwandeln, weiter welche Ursache zu diesen Veränderun. gen führt und auch welche Bedeutung diese Veränderungen haben. Erst dann können wir sagen, dass wir wissen, was Plasmazellen sind.

Die Frage worin die Veränderungen bestehen, die die Zellen zu Plasmazellen umwandeln, halte ich sogar für die wichtigste und interessanteste, um das Wesen der Plasmazellen zu verstehen, viel wichtiger als die Frage, welche Zellarten, ob Bindegewebszellen oder Leukocyten, diese Veränderungen durchgehen. Erst durch Eindringen in die Frage über die Natur dieser Veränderungen können wir - scheint es mir - bestimmen, was wir Plasmazellen nennen oder nicht nennen sollen.

Doch hat man sich bis jetzt damit nur wenig beschäftigt. Joannovics hat allerdings in seiner Arbeit diese Frage berührt. Er hebt hervor, dass Plasmazellen "bei allen jenen pathologischen Processen gefunden werden, bei denen es einerseits zur Einwanderung von Leukocyten und zur Proliferation von fixen Bindegewebszellen kommt, andererseits ein Zerfall von Zellen und dadurch ein Freiwerden ron chromatischer Kernsubstanz besteht". Alsdann fügt er hinzu: „Da drängt sich die hypothetische Vorstellung auf, die Plasmazellen entstehen dadurch, dass junge Zellen und zwar sowohl Bindegewebszellen als lymphoide Zellen diese Zerfallsproducte von Zellen, namentlich ron Kernsubstanz in ihr Protoplasma aufnehmen." Er hat also die Auffassung von einem und demselben Veränderungsprocess (nämlich Aufnahme von Kernsubstanz) in verschiedenen Zellarten (Bindegewebszellen und Leukocyten). Dieselbe Auffassung, dass es sich bei den Plasmazellen um einen einheitlichen Veränderungsprocess in den beiden Zellarten handelt, hat sich während dieser meiner Arbeit auch mir aufgedrängt. Den Process selbst habe ich mir doch ganz anders als Joannovics gedacht. 
Es ist nämlich auffallend, dass in beiden Arten von Plasmazellen, sowohl Unna's als Marschalko's, der Kern gewöhnlich eine excentrische Lage hat. Dieses muss doch etwas bedeuten. Da wir wissen, dass bei Sekretionszellen in dem Activitätsstadium eine Kernverschiebung stattfindet, liegt der Gedanke sehr nahe, auch bei den Plasmazellen ebenfalls einen intracellulären Process so wie in den Secretionszellen anzunehmen. Auch der in beiden Zellarten oft vorkommende centrale Lichthof scheint mir nicht anders gedeutet werden zu können als durch einen intracellulären Process hervorgerufen. Der Protoplasmazerfall, den wir so oft bei Unna's Plasmazellen gefunden haben, ist ja auch ein intracellulärer Process, wahrscheinlich doch von anderer Art als bei nicht degenerirenden Plasmazellen.

Das Studium von Plasmazellen hat also Joannovics $\mathrm{zu}$ einer hypothetischen Vorstellung von einer von aussen kommenden Veränderung gefübrt, mich aber zu einer Vorstellung von einem reinen intracellulären Process. Selbstverständlich will ich diese Vorstellung nur als eine Hypothese aussprechen und im Vergleich mit Joannovics nur in der Hoffnung stellen, dass sie zum Studium dieser interessanten Frage anregen möge.

Eine ähnliche Anschauung wie ich scheint auch Schottländer(16) zu haben. Er sagt nämlich: „Mit anderen Worten brauchen die Plasmazellen nicht als eine besondere Zellgattung angesehen zu werden, sondern es zeigt bei fixen Gewebszellen ebenso wie bei Leukocyten die veränderte Beschaffenheit des Protoplasmas nur eine zunächst progressive Thätigkeit der Zelle an." Also findet man auch hier, so wie bei Joannovics und mir, denselben Gedanken, dass das charakteristische für die Plasmazellen nicht das Hervortreten aus einer gewissen Zellart, sondern eine bestimmte Zellveränderung ist.

Dieser Gedanke hat sich mir noch auf eine andere Weise aufgedrängt. Ich habe in meinen Lupusfällen gesehen, dass in den Fällen, wo Bindegewebsveränderungen überwiegen, die aus Bindegewebszellen hervorgehenden Unna'schen Plasmazellen vorkommen. Wo es aber zu einer reichlichen Leukocytose gekommen ist, ist es doch zu morphologisch ziemlich gleichartigen Veränderungen (stärkere Protoplasmafärbung, excentrische Kern- 
lage, Lichthof) mit den Leukocyten, statt mit den Bindegewebszellen gekommen. Da man sich aber die Aetiologie in den verschiedenen Fällen doch wohl als dieselbe denken muss, so liegt der Gedanke eines gleichen oder wenigstens gleichartigen Veränderungsprocesses in den beiden Zellarten nahe.

Welcher intracelluläre Process in den Plasmazellen stattfindet, darüber müssen uns erst weitere Untersuchungen Aufklärung verschaffen. Zu diesen hoffe ich später Zeit zu gewinnen.

Zum Schluss will ich Herrn Dr. Max Joseph, in dessen Laboratorium jch diese Untersuchungen ausgeführt habe, für das grosse Interesse, womit er meiner Arbeit gefolgt, für die guten Rathschläge, die er mir gegeben, und für seine grosse Liebenswürdigkeit mir gegenüber, meinen herzlichen Dank aussprechen. Herrn Dr. Leon or Michä̈lis, Assistenten am städt. Krankenhaus, Gitschinerstrasse. bin ich ebenfalls herzlichen Dank schuldig für die vielen Winke, die er mir in den Färbungsmethoden gegeben hat. Herrn Dr. Franz Solger, der auch in Dr. Max Joseph's Laboratorium mit Studien über Plasmazellen beschäftigt ist, danke ich ebenfalls für seine Theilnahme an meiner Arbeit.

\section{Literatur.}

1. B a umgarten. Die Rolle der fixen Zellen in der Entzündung. Berl. klin. Wochensch. 1900. pag. 857.

2. B orst M. Neue Experimente zur Fremdkörperheilung. Verh. d. Deutsch. pathol. Gesellsch. II. (München 1899.) Berlin 1900.

3. Hodara. Kommen in den blatbereitenden Organen des Menschen normalerweise Plasmazellen vor? Monatshefte für prakt. Derm. Bd, XXII. 1896.

4. Jadassohn J. Demonstration von Unna's Plasmazellen und von eosinophilen Zellen im Lupus und in anderen Geweben. Verhandl. d. dentsch. Derm. Ges. Leipzig 1891. Arch. f. Derm. u. Syph. 1892. Ergänzh. pag. 58.

5. Ibidem. Bemerkungen zu Unn a's Arbeit über seine Plasmazellen. Berl. klin. Wochenschr. 1893. pag. 222.

6. Ibidem. Letzte Bemerkungen gegen Herrn P. G. U $\mathrm{n} n$ a. Berl. klin. Wochensehr. 1898. pag. 271.

7. JoannovicsG. Ueber das Vorkommen, die Bedeutung und Herkunft der Un $\mathbf{n}$ a'schen Plasmazellen bei verschiedenen pathologischen Processen. Zeitschrift für Heilkunde. 1899. Band XX. pag. 159.

8. Justi $K$. Ueber die U $n n a^{2}$ schen Plasmazellen in den normalen und tuberculösen Granulationen. Inaugural-Dissertation, Marburg. 1897. 
9. K rom pecher E. Beiträge zur Lehre von den Plasmazellen. Beitr. z. pathol. Anat. u. z. allg. Pathol. von E. Z i e g l e r. Band XXIV.

10. M a r c hand $\mathrm{F}$. Ueber die bei Entzündung in der Peritonealhöhle auftretenden Zellformen. Verhandl. der deutsch. pathol. Gesellsch. I. (Düsseldorf). Berlin. 1899.

11. von $\mathrm{M}$ a $\mathrm{rschalko} \mathrm{Th}$. Ueber die sogenannten Plasmazellen, ein Beitrag zur Kenntniss der Herkunft der entzündlichen Infiltrationszellen. Arch. f. Derm. u. Syph. 1895. Band XXX. pag. 3.

12. Ibidem. Zur Plasmazellenfrage. Centralblatt f. allg. Pathol. u. pathol. Anatom. 1899. Band X. pag. 851 .

13. Ibidem. Die Plasmazellen im Rhinoscleromgewebe mit Nachtrag. Arch. f. Derm. u. Syph. 1900. Band LIV.

14. N e i s s r. Bemerkungen zu v. M a r s c halk o's erster Arbeit.

15. Ibidem. Zur Diseussion über Plasmazellen. Arch. f. Derm. u. Syph. 1895. Band XXXI. pag. 387.

16. S c h ottlä nd e r. Ueber Eierstockstuberculose. Jena. Gustav Fis cher. 1897 .

17. van der $\mathrm{S}$ pe ek und $\mathrm{U} \mathrm{n}$ na. Zur Kenntniss der Waldeyerschen Plasmazellen und Ehrlich'schen Mastzellen. Monatshefte f. prakt. Dermat. 1891. Band XIII. pag. 364.

18. Un n a P. G. Ueber Plasmazellen, insb. beirn Lupus. Monatsh. f. prakt. Derm. 1891. Band XII. pag. 296.

19. Ibidem. Ueber die Bedeutung der Plasmazellen für die Genese der Geschwülste der Haut, der Granulome und anderer Hautkrankheiten. Berlin. klin. Wochenschr. 1892 pag. 1240. pag. 222 .

20. Ibidem. Gegenbemerkungen. Berlin. klin. Wochenschrift. 1893.

21. Ibidem. Ueber Plasmazellen. Antikritisches und Methodologisches Monatsh. f. prakt. Derm. 1895. Band XX. pag. 477.

\section{Erklärung der Abbildungen auf Tafel VII u. VIIf.}

Fig. 1. Färbung mit polychromem Methylenblau und Entfärbung in Unn a's Glycerinäthermischung. Vergrösserung $650 / 1$. Das Bild stellt einen Theil eines Lupusknötchens von Fall 1 dar; $a$ ein Theil des blassen aus Epitheloidzellen " bestehenden Centrums, $b$ ein Thesl aus derr ringförmigen Infiltrationswall, $e$ umgebendes Bindegewebe, $g$ ein Gefäss an der Grenze zwischen $a$ und $b$. $U$ Un n a'sche Plasmazellen, $U k$ solche ohne Kernkörperchen, $U r$ solche mit einem Ring von Chromatinkörnchen in der Kernperipherie, Ue solche mit excentrischer Kernlage, Ua solche mit Ausläufer versehenen, $Z$ Zwischenformen zwischen Unn a'schen Plasmazellen und Bindegewebszellen, $M$ Mastzellen, $f$ freie Mastzellenkörner im Gewebe, $b$ Bindegewebszellen.

Fig. 2. Härbung mit polychromem Metbylenblau und Entfärbung in Glycerinäthermischung. Vergrösserung ${ }^{6.50} / 1$ Das Bild stellt einen Theil eines Infiltrationswalles eines Lupusknötchens in Fall 1 dar. $U$ Un na'sche Plasmazellen. $Z Z$ wischenformen $z$ wischen diesen und Bindegewebszellen. $T$ kleine Zellen, die wahrscheinlich Un n a's Tochterplasmazellen entsprechen. $M$ Mastzellen. 
F ig. 3. Färbung mit reinem Methylenblau. Vergrösserung $650 / 1$. Das Bild ist eine Sammlung von $U \mathrm{n} n$ a's Plasmazellen, einem Infiltrationswall, eines Iupusknötchens in Fall I entnommen. $x$ ist eine Zelle, die bei einer gewissen Einstellung der Mikrometerschraube nur einen Lichthof in der Mitte zeigt. $y$ ist dieselbe Zelle bei einer höheren Einstellung, wo man in der Mitte einen Korn sieht.

Fig. 4. Färbung mit polychromem Methylenblau und Entfärbung in Unna's Glycerinäthermischung. Vergrösserung ${ }^{650} / 1$. Das Bild stellt einen Theil des diffusen Granulationsgewebes aus Fall 2 dar. Das Gewebe ist von einem Gefässe $g$ durchzogen. $r$ rothe Blutkörperchen. $l$ Lymphocyten. $p$ polynucleäre Leukocyten. $e$ Endothelzellenkerne. $b$ Bindegewebszellenkerne. $M$ Mastzellen. $P$ von $\mathrm{M}$ a r chalko's Plasmazellen. $Z$ Zwischenformen zwischen $P$ und $l$.

Fig. 5. Färbung mit B ö h m er's Hämatoxylin. Vergrösserung ${ }^{650} / 1$. Das Bild ist ein Theil desselben Granulationsgewebes wie in Fig. $4 P$ von Marschalko's Plasmazellen. l Lymphocyten. $b$ Bindegewebszellenkerne. $g$ ein Gefäss. $e$ Endothelzellenkerne. rothe Blutkörperchen.

Fig. 6. Färbung mit polychromem Methylenblau und Entfärbung

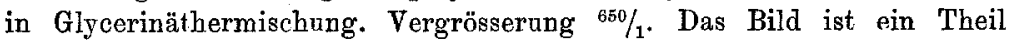
eines Infiltrationswalles eines Lupusknötchen des Falles 5. $b$ Bindegewebszeilen. $d \mathrm{Unn}$ a'sche Plasmazellen mit mehr oder weniger degenerirtem Protoplasma.

Fig. 7. Färbung mit polychromem Methylenblau und Entfärbung in Unna's Glycerinäthermischung. Dann Färbung in Eosin. Vergrōsserung ${ }^{650} / \mathrm{x}$. Das Bild stellt einige Zellen dar, die isolirt im Bindegewebe vom Fall 5 liegen. $U$ eine $U \mathbf{n} n$ a'sche Plasmazelle. $d$ Un $n$ a'sche Plasmazellen mit mehr oder weniger degenerirtem Protoplasma. Die übrigen Zellen sind Bindegewebszellen.

F i g. 8. Färbung mit polychromem Methylenblau und Entfärbung in Unna's Glycerinäthermischung. Vergrösserung ${ }^{650} / 1$. Das Bild zeigt einige Zellen aus einem Infiltrat in dem Bindegewebe des Falles 5. $M$ Mastzellen. $b$ Bindegewebszellen. Die übrigen sind Unna'sche Plasmazellen mit mehr oder weniger degenerirtem Protoplasma.

Fig. 9. Färbung mit reinem Methylenblau. Vergrösserung ${ }^{850} / 1$. Das Bild zeigt eine Gruppe von Un n a'schen Plasmazellen umgeben von einigen spindelförmigen Bindegewebszellen. Stammt aus Fall 5.

Fig. 10. Färbung mit polychromem Methylenblau nnd Entfärbung in Un $n$ a's Glycerinäthermischung. Das Bild stammt aus Fall 6. Es zeigt ein Lymphgefäss $g$ mit einem Proliferationsprocess im Bindegewebe um sich herum. $k$ ist eine Bindegewebszelle in Theilung mit Kerntheilungsfigur. $b$ andere Bindegewebszellen. $U$ typische Un $n$ a'sche Plasmazellen. $d$ solche mit mehr oder weniger degenerirtem Protoplasma. 


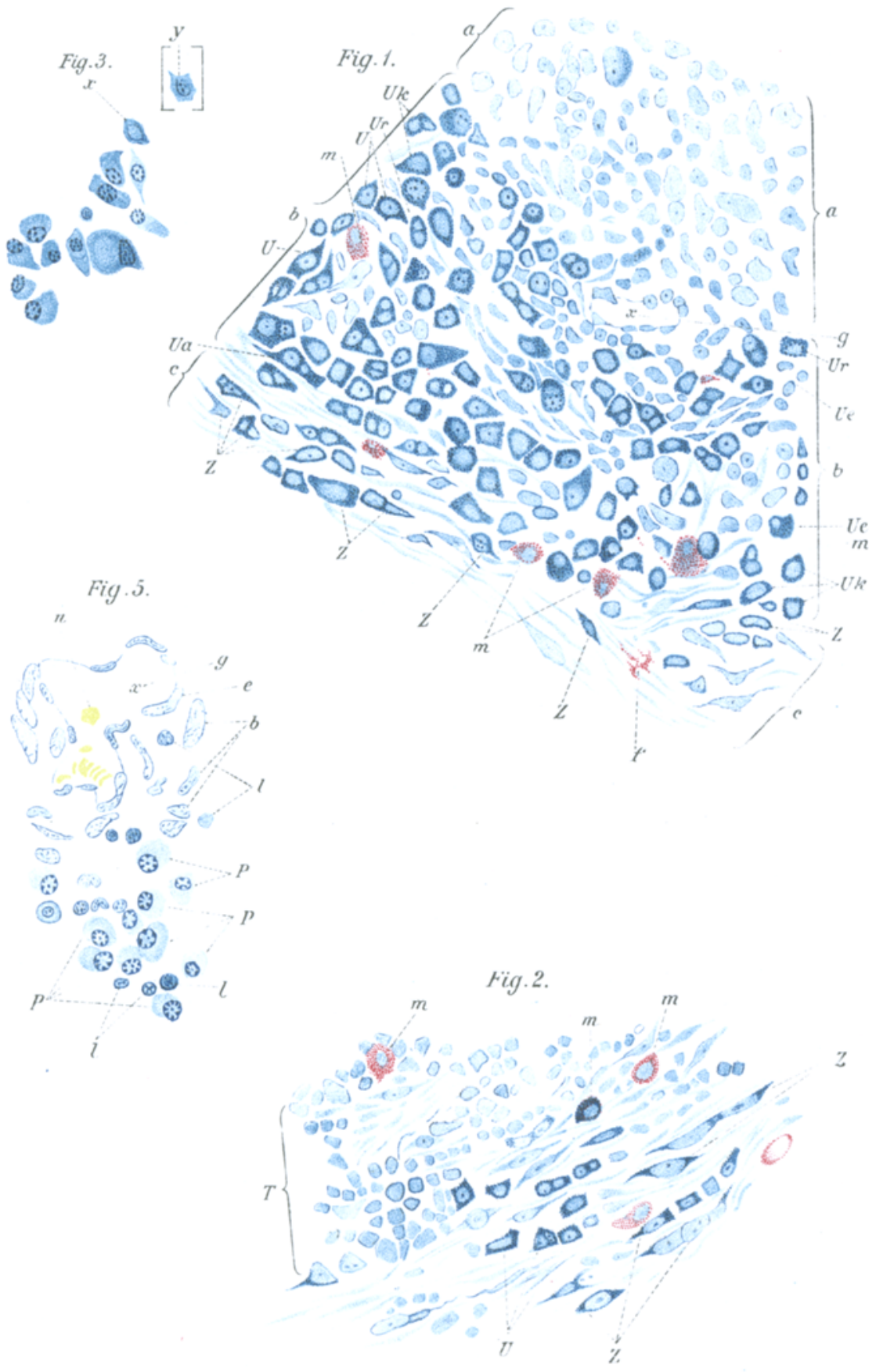

Almqvist: Zur Kenntniss der Plasmazellen. 


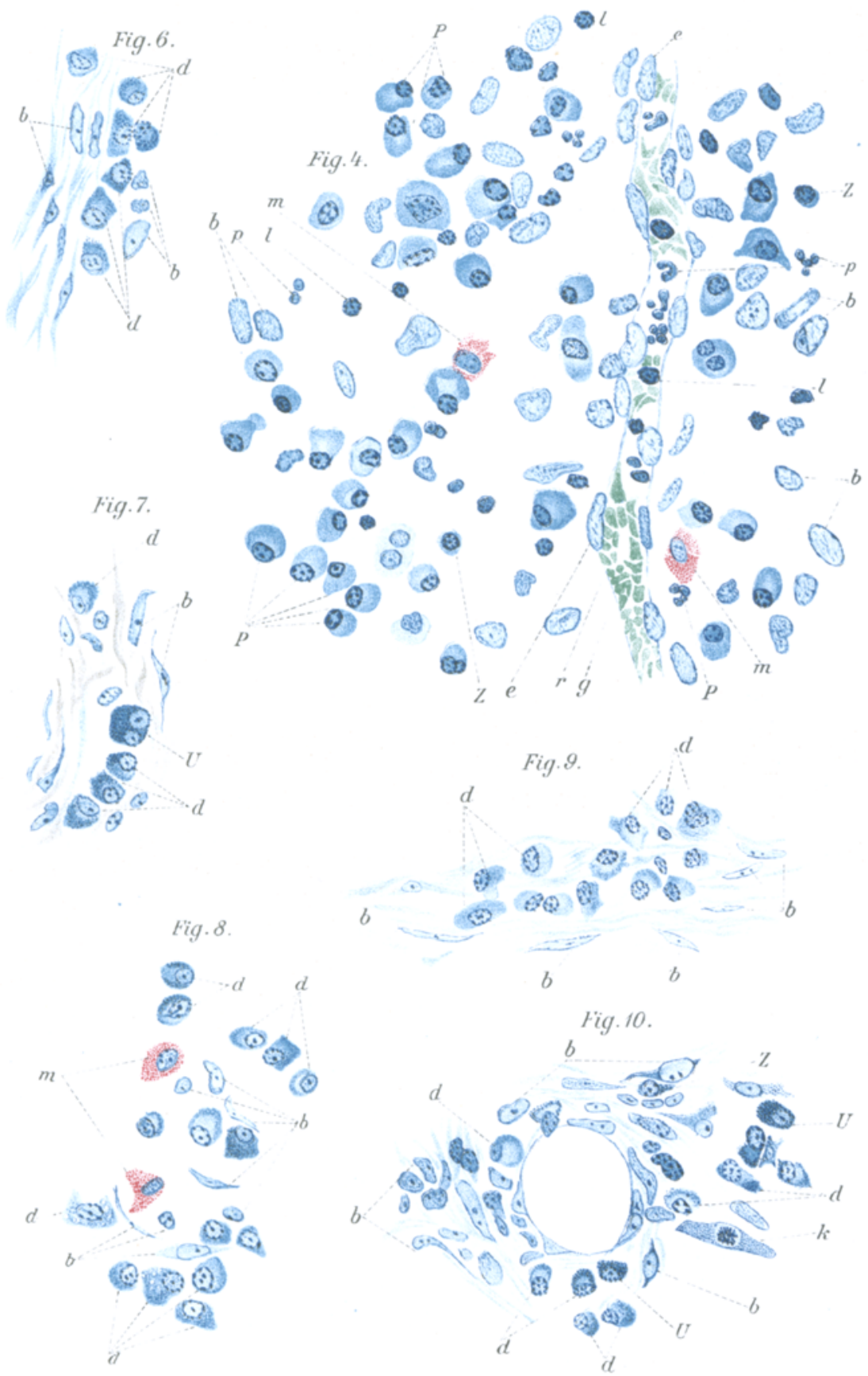

Almqvist: Zur Kenntniss der Plasmazellen. 